\title{
ZUR INTERAKTIONSSTRUKTURELLEN FUNDIERTHEIT VON SPRACHVARIATION ${ }^{1}$
}

\begin{abstract}
Der Aufsatz stellt zuerst ein Projekt zur Standard-Dialektvariation in einer schwäbischen Kleinstadt vor. Dabei wird ganz kurz auf die soziolinguistische Situation des südwestdeutschen Sprachraumes und auf methodische Aspekte der Analyse von Dialekt-Standard-Variation eingegangen. Es folgen Bemerkungen zum institutionellen Setting Gemeinderat bevor an einem Datenbeispiel aus einer Sitzung des Gemeinderats der Einsatz von kontrastbildenden Sprachvariationsverfahren als rhetorische Ressource gezeigt wird. Das sequenzanalytische Vorgehen belegt dabei die interaktionsstrukturelle Fundierung von Sprachvariationsverfahren.

This article first describes a project on standard dialect variation in a small town in Swabia. The sociolinguistic situation in South West Germany and methodological aspects of the analysis of dialect standard variation are discussed briefly. This is followed by remarks on the institutional setting 'local council', leading up to the presentation of example data from a meeting of the local council which show the use of speech variation as a means to produce contrast in order to support rhetorical procedures. The sequence analysis approach employed shows that forms of language variation are based on the structure of the interaction.
\end{abstract}

\section{Sprachvariation in einer schwäbischen Kleinstadt - Varietäten im Diskurs}

Das von Werner Kallmeyer betreute Dissertationsprojekt „Sprachvariation in einer schwäbischen Kleinstadt - Varietäten im Diskurs" behandelt Dialekt-Standard-Variation auf verschiedenen ausgewählten kommunikativen Schauplätzen einer Sprechergemeinschaft im Mittelschwäbischen. ${ }^{2}$ Die primäre Datenbasis besteht aus Tonaufnahmen von sprachlichen Interaktionen im Gemeinderat, den Schulen der Stadt und zwei unterschiedlich orientierten Freizeitgruppen. Ziel der Arbeit ist die Beschreibung des binnensprachlichen Varianzraums ,Kleinstadt in Südwestdeutschland' durch die Analyse der Funktionalität und Relevanz von Sprachvariation für die Sprecher.

\subsection{Aspekte der soziolinguistischen Situation}

Im schwäbischen bzw. gesamten oberdeutschen Sprachraum gelten die Sprecher immer noch als dialektloyal bzw. der Dialekt als vital. ${ }^{3}$ Zur traditionellen Dialektloyalität kommt im untersuchten schwäbischen Standard-Dialekt-Kontaktraum Spannung und Dynamik durch standardsprachorientierte Innovation hinzu. Die Standardsprache - wenn

\footnotetext{
${ }^{1}$ Ich danke Ibrahim Cindark und Reinhold Schmitt für wertvolle Hinweise in Analysesitzungen sowie Stefan Kleiner, Ulrich Reitemeier und Wolfgang Schütte für wichtige Hinweise zum Text.

${ }^{2}$ Die untersuchte Gemeinde Erbach liegt an der Donau, ca. 13 Kilometer westlich von Ulm. Sie hat sich seit Ende des Zweiten Weltkriegs von einer ziemlich homogenen Dorfgemeinschaft zur Kleinstadt im Einzugsbereich der Stadt Ulm entwickelt.

${ }^{3}$ Siehe bspw. die Einschätzungen zum Oberdeutschen in Gilles (2003) oder Mihm (2000). Sicherlich muss bei einer angenommenen, relativen Vitalität' mit Unterschieden zwischen städtischen und ländlichen Räumen (bzw. Zentrum und Peripherie) gerechnet werden.
} 
auch ein regional transformierter und deliteralisierter Standard - erobert Sprechdomänen bzw. darin Funktionsbereiche.

Dieser Untersuchungsraum kann aus soziolinguistischer Sicht beschrieben werden als komplexe Kontaktsituation, in der die Sprecher die Wahl haben zwischen koexistierenden Formen innerhalb ihres linguistischen Repertoires, das sich aus mehr oder weniger diffusen Varietäten bzw. systemoiden Verdichtungen von Varianten zusammensetzt. ${ }^{4}$

Im Zentrum der Analyse steht die interaktionsstrukturelle Einbettung von Sprachvariation - allerdings nicht nur aus konversationsanalytischem Interesse allein, sondern auch um formbezogene Fragen zum Varietätengefüge bei Sprechern im südwestdeutschen Untersuchungsraum zu behandeln. Die interaktionsstrukturellen und funktionalen Eigenschaften werden $\mathrm{zu}$ formalen Eigenschaften und Einheiten der gebrauchten variablen Sprachformen in Beziehung gesetzt, um Hinweise zu bekommen auf den kommunikativen Status der linguistischen Varianten und auf sprachstrukturelle Aspekte der verwendeten Repertoires, die gegebenenfalls aus rekurrenten Mustern des Gebrauchs emergieren. Durch die Analyse der Zusammenhänge zwischen Interaktionsstruktur und Formengebrauch können aktuelle dialektologisch-variationslinguistische Probleme behandelt werden, nämlich das Problem der Segmentierbarkeit der Repertoires innerhalb eines formalen Kontinuums, wie es für den oberdeutschen Sprachraum insgesamt konstatiert worden ist (bspw. in Bellmann 1983 u. 1997 oder Auer 1986 u. 2004). Damit hängt die Frage zusammen, was eigentlich ein Kode oder gar eine potentielle Varietät ist (siehe dazu Alvarez-Cáccamo 1990). Ich selbst folge der Perspektive, Hinweise auf den Status von linguistischen Formen und auf die Architektur des Repertoires im Gebrauch durch die Sprecher selbst zu suchen: Elemente eines Kodes oder einer Varietät müssen als solche durch die Sprecher gebraucht bzw. interpretierbar gemacht werden (siehe Auer 1986, S. 97).

\subsection{Bemerkungen zur Methodologie}

Man kann die Forschungsparadigmen zur Beschreibung von Standard-Dialekt-Kontaktsituationen idealtypisch zwei Lagern zuordnen, einerseits den quantitativ ausgerichteten Ansätzen in der Tradition der variationslinguistischen Forschung Labovs und andererseits den qualitativen Beschreibungsverfahren, die aus interaktionsfokussierenden Ansätzen mit phänomenologisch-konstruktivistischer Grundannahme hervorgehen. ${ }^{5}$

Eine Grundlage qualitativer Ansätze ist die ethnomethodologische Konversationsanalyse, die verbunden ist insbesondere mit den ,Kaliforniern' Garfinkel, Sacks und Schegloff sowie in der europäischen Rezeption und Weiterführung mit dem Namen Werner Kallmeyer. Daneben gehören die Ethnographie des Sprechens (nach Hymes und Gumperz) und die interpretative Soziolinguistik Gumperzscher Prägung zum qualitativen Paradigma.

Auf Unterschiede zwischen den Lagern wird im Folgenden nicht eingegangen, sondern lediglich festgestellt, dass deutsche Standard-Dialekt-Kontaktsituationen bislang domi-

\footnotetext{
${ }^{4}$ Siehe dazu die Konzepte „Verdichtungspunkte“ (Berruto 1987, S. 265), „Verdichtungsbereiche“ (Lenz 2003, S. 32 u. 250) bzw. „Sprechlagenschwerpunkte“ (Schmidt 2005, S. 70).

${ }^{5}$ Gilles (2003) fasst die Ansätze der beiden Paradigmenlager treffend unter den Begriffpaaren ,korrelativglobal“ (quantitativ) und „konversationell-lokal“ (qualitativ) zusammen.
} 
nant aus korrelativ-globaler Perspektive bearbeitet worden sind. Eine der wenigen Ausnahmen ist das IDS-Projekt „Kommunikation in der Stadt“, bei dem Methoden der qualitativen Ansätze verbunden worden sind zur ethnographischen Gesprächsanalyse, um neben anderen Gesprächsphänomenen auch binnensprachliche Variation zu untersuchen. ${ }^{6}$

Bei der Untersuchung des Variationsraumes schwäbische Kleinstadt wird ein integrativer Ansatz versucht, d.h. die Verbindung von qualitativen und quantitativen Methoden. Es werden einerseits tatsächliche Kommunikationspraktiken der Sprecher und insbesondere innersituative Sprachvariation untersucht. Das Verhalten der variablen sprachlichen Formen wird in Bezug gesetzt zu kommunikativen Funktionen und interaktionalen Aufgaben der Sprecher auf den verschiedenen Ebenen der Interaktionskonstitution (Kallmeyer 1978 u. 1982). Andererseits werden die Variablen-Varianten-Verteilungen bei den untersuchten Sprechern quantitativ erfasst, um Hinweise zu bekommen auf die individuellen Formenspektren bzw. linguistischen Profile der Sprecher. Das linguistische Profil ergibt zusammen mit Kovariations- und Kookkurrenzmustern entscheidende Hinweise auf das Variationsprofil bzw. Variationstypik und damit die Grundlage dafür, die situativen Normallagen der Sprecher fassbar zu machen. Normallage bzw. Normalformerwartungen sind Elemente des von den Teilnehmern wechselseitig als geteilt unterstellten dynamischen Interaktionswissens und zentraler Bezugsrahmen bei der Interpretation der konkreten Variationsverfahren als Kontextualisierungshinweise (Cook-Gumperz/Gumperz 1976; Gumperz 1982).

\section{Schauplatz ${ }^{7}$ Gemeinderat}

Der Gemeinderat ist in mehrfacher Hinsicht für mein Untersuchungsinteresse geeignet. Es handelt sich dabei um eine funktionsbezogene soziale Welt mit regelmäßigen Treffen, in der Kommunikation eine wesentliche Rolle spielt. ${ }^{8}$ In der institutionalisierten sozialen Situation findet das Reden im Spannungsfeld von strukturellen Vorgaben bspw. durch die Agenda, die Rednerliste (one-slot-Prinzip) oder durch unterschiedliche Handlungsrollen - und individuellen Gestaltungsmöglichkeiten statt. Potentiell interaktions- und sprachwahlimplikativ sind dabei folgende Faktoren:

1) Die Zwiespältigkeit der Funktion des Gremiums an sich: Die Funktionalität des Gemeinderats spaltet sich grundsätzlich auf in einerseits die konkrete Verantwortung für

\footnotetext{
${ }^{6}$ Siehe zu „Kommunikation in der Stadt“ Kallmeyer/Keim/Nikitopoulos (1982), Kallmeyer (1994), Keim (1995) und Schwitalla (1995). Siehe zur Verbindung von ethnographischen und gesprächsanalytischen Methoden Deppermann (2000).

${ }^{7}$ Ich stützte mich auf Ergebnisse des Projekts „Kommunikation in der Stadt“; zentrale Konzepte wie „Schauplatz“, „,Soziale Situation“, „,Soziales Ereignis“ und „,soziale Welt“ werden im Folgenden so verwendet, wie sie dort verwendet bzw. entwickelt worden sind. Siehe dazu Kallmeyer/Keim/Nikitopoulos (1982, S. 354-362) sowie Schwitalla in diesem Band.

${ }^{8}$ Die Tatsache, dass es in jeder Gemeinde eine vergleichbare Kommunikationssituation gibt, macht den Schauplatz Gemeinderat auch interessant für großflächige dialektologisch-dialektgeografische Untersuchungen zur Variation im Raum; auf Grund des relativ hohen globalen Formalitätsgrades der Sitzungen ließe sich insbesondere die regionale Variation des am kodifizierten Standard orientierten Sprechens erfassen (siehe Lameli 2004).
} 
die Sicherung und Versorgung der Stadt und andererseits in die symbolische Funktion der Repräsentation der Gemeinde nach innen wie nach außen (Luckmann 1970).

2) Mehrfachadressierung (Kühn 1995): Beim Sprechen treten die Teilnehmer einerseits in direkte Handlungsbeziehungen zueinander und zugleich in eine Beziehung zur Öffentlichkeit, die selbst wieder mindestens zwieschlächtig ist: Sie ist repräsentiert einerseits durch die beisitzenden Gemeinderäte und das kopräsente Publikum und andererseits durch Journalisten der beiden relevanten Tageszeitungen. Die Mehrschichtigkeit der Adressierbarkeit ist die Basis für sogenannte Fensterreden bzw. für das Phänomen der Trialogizität (Dieckmann 1981).

3) Die Teilnehmer im Gemeinderat interagieren nicht losgelöst von übergeordneten soziolinguistischen Orientierungen. Das betrifft insbesondere die komplexe Prestigesituation der Standard- und Dialektformen im Untersuchungsraum. Hier will ich an eine metasprachliche Äußerung eines Mannheimer Informanten des Stadtprojekts erinnern, die die Komplexität der Sozialprestigezuschreibung von Sprachformen verdeutlicht und inhaltlich ähnlich auch im Schwäbischen denkbar ist: „unser schbroch is a e schbroch“ (Kallmeyer 1996b).

Bei den sprachlichen Interaktionen im Gemeinderat fällt eine grundlegende Doppelorientierung der Sprecher auf. Einerseits sind die Sitzungen geprägt von einer kommunikativen Arbeits- und Ergebnisorientierung. Das Sprechhandeln folgt weitgehend einem relativ festen Rahmen von etablierten Regeln. Die ideale Leitnormvorstellung des Rates sieht aufgaben- bzw. themenorientiertes Reden vor auf der Grundlage einer insgesamt sehr konsensuellen Ausrichtung; die Orientierung an einer übergeordneten kooperativen Interaktionsmodalität sichert das gemeinsame Suchen nach Entscheidungen zum Wohle der Gemeinschaft. ${ }^{9}$ Andererseits bietet sich der Schauplatz den Teilnehmern als Bühne der strategischen Auseinandersetzung an, auf der sie mit rhetorischen Verfahren versuchen, an ihrem Status, Autorität und Prestige zu arbeiten. Es gehört zur etablierten Erwartenserwartung, dass gezeigt wird, dass Politik gemacht wird und Räume zur Darstellung und Positionierung als Politiker genutzt werden. Selbst auf der basalen politischen Ebene geht es um symbolisches Kapital. ${ }^{10}$ Die im untersuchten Sprachraum allgegenwärtige Standard-Dialekt-Variation kann für einige Lokalpolitiker zur Unterstützung ihrer rhetorischen Verfahren gebraucht werden, die dann in den meisten Fällen zusammen mit anderen Ressourcen konkomitant eingesetzt wird.

Die Variationsmuster im Gemeinderat sind aufgrund seiner relativ heterogenen Zusammensetzung $^{11}$ sehr abhängig vom linguistischen Profil der Sprecher im Sinne des linguistischen Spektrums auf der Grundlage von Sprecherkompetenz und Präferenzen, das die verschiedenen Sprecher in die Situation einbringen, und das durch die Situation nicht so stark transformiert wird, dass es nicht im Variationsprofil erkennbar bliebe.

9 Die von den Sprechern geteilte Normalform und Erwartung ist erkennbar an Indikatoren der Geltung einer „preference for agreement“ (Sacks 1987), an selbst- und nötigenfalls fremdinitiierten Beschränkungs- und Regulierungsmaßnahmen bezüglich der Art und besonders Länge der Äußerungen sowie Fokussierungsverfahren mit präliminaren Elementen zur Äußerungslegitimierung und -modalisierung.

${ }^{10}$ Insbesondere in Episoden, in denen die Arbeitsorientierung zugunsten der strategischen Interaktion in den Hintergrund gerät, kann sich auch die Ausrichtung an etablierten Ordnungsstrukturen der Interaktion verändern. Bspw. kann sich im Kontext strategischer Interaktion die Präferenzordnung umkehren zur ,preference for disagreement“ (Bilmes 1991).

11 Lameli (2004) spricht von ,teilhomogener Zusammensetzung““ 
Auf die Profile der im folgenden Beispiel beteiligten Sprecher wird hier nicht näher eingegangen. Es wird lediglich darauf hingewiesen, dass die beiden Sprecher über ein weites Formenspektrum verfügen, das sie typischerweise shiftend-switchend ${ }^{12}$ bis mischend verwenden, also im Sinne von Peter Auers Typologie (Auer 1999) im Übergangsbereich zwischen Code-Switching und Language-Mixing. ${ }^{13}$

\section{Beispiel Müllgebühren}

Im ausgewählten Ausschnitt aus dem Verwaltungsausschuss der Stadt bietet sich den Gemeinderäten eine Gelegenheit zum politischen (strategischen) Reden. Der Agendapunkt, den es im Ausschuss für eine Entscheidung in der nächsten Gesamtratsitzung vorzubereiten gilt, ist den Beteiligten klar - in ihren eigenen Worten ,bleibt nichts anderes übrig' bzw. ist der Beschluss des Verwaltungsvorschlags ,unumgänglich'. Lediglich der Prozess, wie man an diesen Punkt gekommen ist, kann kritisiert werden.

Konkret geht es im Datenausschnitt um das Problem, dass ein Beschluss des Gemeinderats offiziell zurückgenommen werden muss. Es war eine Woche vor der hier präsentierten Sitzung des Verwaltungsausschusses entschieden worden, die Müllgebühr für die Verbraucher zu senken. Am Tag nach dieser Entscheidung hat allerdings der Kreistag die Müllgebühren für die Kommunen des Landkreises erhöht; d.h. die Stadtverwaltung muss die nicht erwarteten und kalkulierten höheren Kosten an die Verbraucher in der Gemeinde weitergeben. Die schon öffentlich gemachte Entscheidung der Gebührensenkung muss zurückgenommen werden, um keinen Verlust zu machen. Das Problem bei der Entscheidungsrücknahme ist die damit verbundene Imagebedrohung für den Gemeinderat als politische Institution; die Rücknahme vermittelt nach außen nicht den Eindruck professioneller politischer Arbeit. Diesen Punkt kritisieren zwei Gemeinderäte und werfen der Verwaltung und insbesondere dem Bürgermeister vor, nicht rechtzeitig über die anstehende Gebührenerhöhung des Kreises informiert gewesen zu sein. Der Bürgermeister ist gleichzeitig Chef der Verwaltung und Mitglied des Kreisrates.

Zur Grobstruktur der Behandlung des Agendapunkts: Nach der einleitenden Problempräsentation durch den Bürgermeister referiert ein Verwaltungsangestellter gemäß institutionalisiertem Ablaufmuster den Sachverhalt mit der von Verwaltungsseite gegebenen Abstimmungsempfehlung. Dem Referenten der Verwaltung folgt der erste Kritiker (K1), auf den der Bürgermeister direkt reagiert. Dann kommt ein Beitrag eines Gemeinderats, der nur kurz und nicht kritisch auf das Problem eingeht, dem allerdings ein zweiter kritischer Beitrag (K2) folgt.

Ich möchte im Folgenden auf die Struktur der beiden kritischen Beiträge eingehen. Aus Platzgründen muss ich davon absehen, auf den vorgängigen Kontext und insbesondere auf die Problemrepräsentation durch den Bürgermeister Bezug zu nehmen.

\subsection{Kritiker 1}

Der erste Kritiker (K1) beginnt seinen Beitrag mit einer Äußerung, die in nuce enthält was er in seinem Beitrag sagen will:

\footnotetext{
${ }^{12}$ Was man nach Lenz (2003, S. 397) „swiftend“ nennen könnte.

${ }^{13}$ Zur Anwendbarkeit der Typologie in der binnensprachlichen Kontaktsituation siehe Knöbl (i. Dr.).
} 
also i find ne to"lle leistung war dəs nicht $\uparrow$ liebe verwalt ${ }^{h} u n g \uparrow$.

Die rhetorisierte, litotisch formulierte und in der Adressierung ironische Bewertung hat Projektionswirkung auf den gesamten Beitrag und ist Rahmenelement für drei folgende Redeteile: ${ }^{14}$

1) Die erste Sequenz kann aktivitätsbezogen als Bewertungssequenz bezeichnet werden (Partiturfläche [1]-[8] im Transkript ${ }^{15}$ ). Sie enthält:

a) die einleitende Wertung

also i find ne to"lle leistung war dos nicht $\uparrow$ liebe verwalt'ung $\uparrow$,

b) eine sehr detaillierte Beschreibung des behandelten Problems

uns hier- *äh n vorschlag zu machn gebühron zu senken- * un am glarchn tag beschließt- *äh do

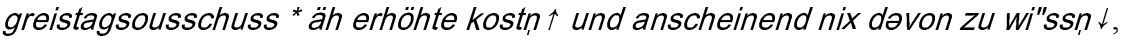

davon kodierniveal abgesetzt

c) einen impliziten Vorwurf

also da frag i mI scho $\uparrow$ * fü was he=mə eiplich en bürgermeischder im greisdag sitzṇ $\uparrow$ *

und explizierten Vorwurf

äh der muss doch informiert sein über solche di"nge- dəs würd i einfach erwardṇ $\uparrow$ drum- * >si=mə

ja eiplich au froh $\uparrow$ dass do bü"rgermeischder< in solchan gremien i"schd $\uparrow$ *

sowie

d) eine Unmutsäußerung bzw. subjektivierte Bewertung

und äh also * so"lche dinge die gfallat mir ü"berhaubt nicht $\uparrow$ muss i scho sagn-.

Die Bewertungssequenz schließt mit

e) einer Wiederholung der einführenden Bewertung

dəs isch au ne- * kor übermäßig tolle leischdung $\downarrow$ *

2) Der Bewertung folgt als zweiter Redeteil eine ausführliche Belegliste mit expliziten Referenzen, die die argumentative Grundlage für die vorgängige Bewertung und nachfolgende Forderung liefert:

äh denn- $m \rightarrow i$ moIn $\leftarrow$ solche dinge ** äh ä da müssn vo"rinformationen da sein-

də äh landrats/ oder də landkreis- * der äh * macht dəs ja nicht so" überraschnd-

$\rightarrow$ da sind ja sitzungsvorlagn, draußn- $\leftarrow$ * da muss me doch zugang habn,-

insbesondre die verwaltung muss zu solchon dingn zugang habn $\downarrow$ * [8]-[11].

Formal auffällig ist die für argumentative Listenaufzählungen typische Rhythmisierung und projektive Intonation. ${ }^{16}$ Zudem fällt das relativ konsistente Kodierniveau der ge-

${ }^{14}$ Die einleitende Bewertung bearbeitet als „Fokussierung“ die Aufmerksamkeitsausrichtung in der Art einer ,vorgreifenden Verdeutlichung“ (Kallmeyer 1978, S.194 sowie S. 229-239).

${ }^{15}$ Die Transkripte der beiden Sprecher befinden sich im Anhang. Die Interaktionen sind nach IDS-Konventionen transliteriert worden (siehe Anhang). Zudem werden die lautlichen Realisierungen der fokussierten Variablen durch IPA-Zeichen repräsentiert, wenn die genaue Darstellung und Unterscheidung (und somit auch die quantitative Erfassung) mit den Laut-Graphem-Korrespondenzen der alphabetbasierten Transliterationsregeln nicht möglich ist. Die hybride Transkriptionsweise ist als Kompromiss zwischen einer möglichst einfach lesbaren Darstellung und der nötigen genaueren entwickelt worden.

${ }^{16}$ Die Listen-Rhythmik ist durch die Parallelstruktur der Intonationsphrasen geprägt - allesamt kurze eigenständige Turnkonstruktionseinheiten (turn construction units), deren letzte Akzentsilben auf ein ähnlich hohes F0-Niveau angehoben sind, das mehr oder weniger bis zum jeweiligen Einheitenende 
brauchten linguistischen Formen auf, die man fast alle dem regionalen Sprechstandardbereich zuordnen $\operatorname{kann}^{17}$, und die mit dem Rahmenelement $\rightarrow i$ moIn $\leftarrow$ sowie mit dem vorausgehenden Ende der Bewertungssequenz kontrastieren.

3) Die Schlusssequenz ,Konsequenz / Forderung', wobei zwischen zwei Forderungsformulierungen innerhalb eines Wenn-Dann-Formativs eine letzte Schuldzuweisung eingeschoben ist:

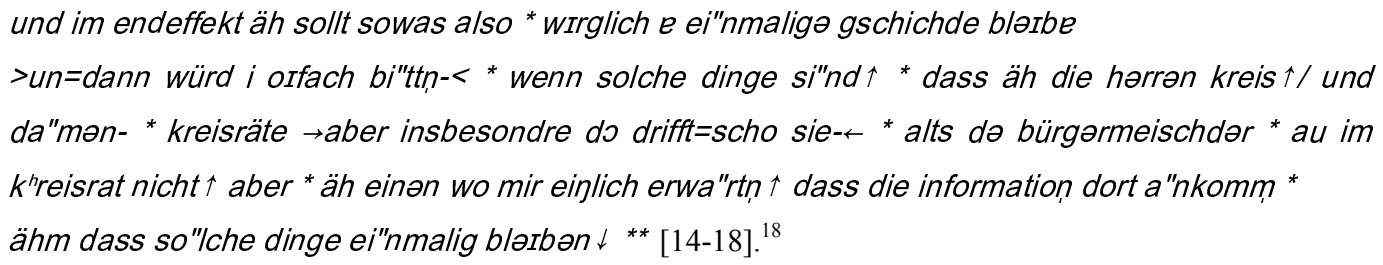

Die drei herausgestellten Redeteile enthalten alle den zentralen Informationsvorwurf, allerdings in jeweils unterschiedlichen Formulierungsformaten und mit variierenden Adressierungen und Referenzen auf den bzw. die Schuldigen:

gehalten wird (siehe zu „Plateau-Konstruktionen“ in offenen Listen Selting 2004, S. 24. Die weiterweisende Wirkung des Plateaus ist im Transkript angezeigt durch den Querstrich als Symbol für ,schwebende Intonation'). Die prosodische Parallelität wird durch zumindest partielle syntaktische Parallelität (durch die Repetitiv-Struktur da müssn .../ da sind .../ da muss ...) unterstützt, die den Eindruck der Gestaffeltheit und Offensichtlichkeit der Argumentationsfolge erhöht. Der fallende Abschluss der letzten Intonationseinheit (... zugang habn $\downarrow$ ) unterstützt den apodiktischen Schluss der Argumentation. Zudem benutzt der Sprecher einen Trick: Er produziert eine typische dreigliedrige Listenkonstruktion mit Detaillierungsprojektion (solche dinge), (offener) Liste und abschließender Gestaltschließung (Selting 2004). Allerdings bindet er in die prosodisch und syntaktische Parallelstruktur - an letzter Listenposition, die oft durch ein zusammenfassendes bzw. übergeordnetes Elemente besetzt wird (,generalized list completer", Jefferson 1991) - ein Element ein (da muss me doch zugang habn-), das semantisch nicht mit den vorgängigen Listenelementen kongruiert und eigentlich zur Post-Detaillierungskom-ponente gehört. Zur Gestaltschließung mit typischer fallender Tonhöhe wiederholt K1 die Schlussfolgerung (insbesondre die verwaltung muss zu solchən dingn zugang habn $\downarrow$ ), wobei er durch die Wiederholung zudem die Subjekt-Referenzen der beiden Konklusionssätze kontrastieren kann.

${ }^{17}$ Bei den Realisierungen der Funktionswörter $<$ das $>$ und $<$ man $>$ als [dəs] und [me] ist eine grobe Zuordnung zum Dialekt- oder erweiterten Standardbereich sehr schwierig. Beide Formen stehen hier in Nebentonpositionen, in denen im Deutschen generell „satzphonetische“ Reduktionen rhythmusbedingt auftreten (Kohler 1995, S. 116 u. 215). Trotzdem entsprechen die beiden Reduktionsformen nicht unbedingt allgemein-deutschen Schnellsprechregeln, sondern treten eher räumlich begrenzt auf (im Oberdeutschen). Die dialektale Reduktionsform unterscheidet sich von der dialektalen Vollform im Fall des $<$ das $>$, nämlich [dəs] vs. [de:s] (mit langem geschlossenem e). Dagegen kann damit gerechnet werden, dass $<$ man $>$ im Dialekt normalerweise sowohl in Akzent- wie auch in Nebentonposition mit n-Elision realisiert wird.

${ }^{18}$ Genau genommen gehört eine Passage zwischen Belegliste und abschließender Forderung zur Schlusssequenz. In [11-14] formuliert K1 mögliche Sichtweisen auf Wirkung und Konsequenz des Vorfalls, nämlich aus potentieller Bürgerperspektive (abril abril und rolle rückwärts), Verwaltungsperspektive (mit einem Zitat aus der Problempräsentation des Bürgermeisters: wär zu schön gewesn) und aus seiner Perspektive, mit der er die Verwaltungssicht und insbesondere die Präsentationsstrategie behandelt (alles schön verbrämt ...). 
In Sequenz 1 (Bewertung) ist der Vorwurf der genauen Situationsbeschreibung angehängt als Schlusselement eines logikorientierten Formats und ohne Referenz auf Schuldige:

und anscheinend nix dovon zu wi"ssn $\downarrow$ [3].

Mit der Situationsbeschreibung kommentarartig verknüpft durch ,also' ist der indirekte und dann ausformulierte Vorwurf mit Schuldreferenz auf die Funktionsrolle des Bürgermeister in dritter Person:

also da frag i mi scho ${ }^{*}$ * fü was he=mə einlich en bürgermeischder im greisdag sitzṇ ${ }^{*}$ äh der muss doch informiert sein über solche di"nge- dos würd I einfach erwardṇ $\uparrow \ldots . .3-6]$.

In Sequenz 2 (Belegpassage) ergibt sich der Vorwurf als Schlussfolgerung aus der Belegliste mit generellem, man' und im Wiederholungskontrast, die Verwaltung' als Schuldreferenzen:

da muss me doch zugang habn- insbesondre die verwaltung muss zu solchon dingn zugang habn $\downarrow[10-11]$.

Zum Schluss in Sequenz 3 (Forderung) ist der Vorwurf am explizitesten und direkt an den Bürgermeister als Funktionsträger adressiert. Beachtenswert ist dabei die Pause $(*)$ zwischen der persönlichen Adressierung und dem relativierenden Verweis auf die Funktionsrolle:

$\rightarrow$ aber insbesondre do drift=scho sie- * alts do brrgermeischdor * au im kreisrat nicht $\uparrow$ aber *äh einen wo mir einlich erwa"rtn $\uparrow$ dass die information dort a"nkomm [16-17].

Hinsichtlich der Standard-Dialektvariation fallen bei Kritiker 1 inbesondere reliefgebende Dopplungen auf. Prominent platziert, nämlich am Anfang und Ende des Beitrags, stehen Formulierungen, die den Kern der „Bewertung“ einerseits und der „Forderung“ andererseits enthalten:

ne to"lle leistung war dos nicht $\uparrow$

am Anfang und

dass so"lche dinge ei"nmalig bleibən $\downarrow$

am Ende. Beide Formulierungen haben allerdings Zweit- bzw. Erstformulierungen in redetextstrukturierender Funktion im Binnentext, nämlich dos isch au ne- * Kor übermäßig tolle leischdung $\downarrow[7-8]$,

die das Ende der Bewertungssequenz markiert und im endeffekt äh sollt sowas also * wrrglich e əI"nmaligə gschichde bləIbe [14]

am Anfang der abschließenden Forderung. Bei beiden Binnentextformulierungen sind im Kontrast zu den prominent platzierten Formulierungen bestimmte linguistische Variablen verändert. ${ }^{19}$

\footnotetext{
${ }^{19}$ Es sind hier die Variablen $<$-st(-)> und <-en(-)>, also die Regionalmerkmale Palatalisierung von $\mathrm{s}$ in nicht-initialer Stellung vor t (bzw. bilabialem oder dental-alveolarem Plosiv) und n-Apokope im Silbenauslaut. Hinweise auf den Status der einzelnen Varianten ist ein Ziel der Arbeit. Es deutet sich allerdings schon an, dass gerade diese beiden Variablen hinsichtlich potentieller Index- oder Salienzwerte zentral sind im untersuchten Raum; siehe zu ihrer Verwendung in einer Schulklasse Knöbl (i. Dr.).
} 
Die Doppelformulierungen lassen zum Einen eine wesentliche Funktion von Sprachvariation erkennen, nämlich die rede- bzw. gesprächsorganisierende Funktionsdimension durch Kontrast (Kallmeyer/Keim 1994). Durch weitere gleichgerichtete Variationsbelege im Korpus könnte zum Anderen geprüft werden, inwieweit die Tatsache, dass die prominenten Positionen jeweils mit Standardformen besetzt sind, ein Hinweis ist auf eine mögliche symbolische Dimension der kontrastiven Formenwahl, nämlich auf weniger emergente als vielmehr gut etablierte, quasi code-inhärente Wertigkeiten wie bspw. mit Standard- und Dialektformen assoziierte unterschiedliche Annahmen von Autorität, Prestige oder Öffentlichkeitsbezug. ${ }^{20}$

\section{$3.2 \quad$ Kritiker 2}

Der zweite Kritiker (K2) geht, nachdem er auf den hier ausgelassenen Vorredner eingegangen ist, in [22] auf das problematische Thema ein. ${ }^{21}$

Die inhaltlich gleiche Ausrichtung wie Kritiker 1 deutet er schon bei der Fokussierung des Themas an, indem er durch die indirekt formulierte Perspektivenübernahme ein Inferenzangebot gibt:

aber ich möcht au noch jetzt äh * bissle zum herr läh/ rübərschaue [22-23].

Damit bezieht sich der zweite Kritiker auf die vorgängige Äußerung des ersten Kritikers und weist diese als relevanten, rahmengebenden Kontext aus, der - so die Behauptung in äußerungskonstitutiver einschließlich kodiernivealer Weise wirkt.

Die der Fokussierung folgende Vorfallsbewertung und Vorwurfsformulierung ist bei Kritiker 2 indirekt präsentiert durch den angeführten Gemeinplatz:

wenn do volksmund sagth es isch dumm gelau"fin- ** dann isch dos meischd!ns soll dos vadeggn- *

dass es vielleicht doch Irgndwelche gibt $\uparrow$ die: äh daran e gewisse schu"ld tragn $\downarrow$ [23-25].

Die bekannte Formel vermittelt den Anspruch allgemeiner Gültigkeit. Der zweite Kritiker bindet die Bewertung nicht wie der erste an die konkrete Situation, sondern nutzt, dass die behandelte Situation vorher von Kritiker 1 genau beschrieben worden ist, um sich mit seiner allgemeinen Formulierung an dessen Thematisierung dranzuhängen. Gerade in der verallgemeinerten Formulierung kann Kritiker 2 sehr deutlich die Frage der Schuld thematisieren. Die Antwort bzw. die konkrete Schuldreferenz ist an dieser Stelle pragmatisch längst klar.

Eine implizite Bewertung und Vorwurf steckt auch im darauf folgenden Übergriff:

äh * soweit * möcht ich jetz net gee zu sage da sieht me wie=s isch wenn d froIe wehler koI grei"sdagsmandat hent $\downarrow[25-26]$.

Der Übergriff ist nicht nur spaßmodalisierte und damit legitimierte Parteipropaganda, sondern bewertet über die Kontrastfolie ,freie Wähler ohne Kreistagsmandat' die betei-

\footnotetext{
${ }^{20}$ Die schwierige Frage hierbei ist, welches ,Bedeutungspotential' innerhalb der Interaktion hervorgebracht, und welches schon konversationsemanent besteht und in Gespräche eingebracht werden kann (siehe dazu auch Hinnenkamp 1987 und Auer 1992, S. 26 oder 1998 S. 154 u. 261.) Die Frage betrifft die Diskussion um Konzepte wie we-code und they-code (bspw. Gumperz 1982, zur Diskussion Sebba/Wootton 1998) und die Beziehung zwischen sprachlichen Kodes und rights and obligations (MyersScotton 1993, zur Diskussion Wei 1998). Hinweise auf etablierte Relationen zwischen Formen/Kodes und Indizierung-/Symbolisierungspotential kann die Untersuchung der stabilen oder flexiblen Direktionalität von Wechseln der Kodierebene bei vergleichbaren Interaktionszusammenhängen geben (siehe dazu Auer 1984, S. 95 sowie 1995, S. 124).

21 Siehe Transkript „Zweitbezug“ im Anhang.
} 
ligten Akteure mit Kreistagsmandat (und insbesondere den Bürgermeister, der zudem als Verwaltungschef für den Gebührensenkungsvorschlag verantwortlich ist). Durch die Ausformulierung der Potenzialität im Fall der freien Wähler behandelt Kritiker 2 die Faktizität beim Bürgermeister mit. Ohne die explizite Erstreaktion durch Kritiker 1 als Kontext wäre die kontrastive Thematisierung zu vage gewesen.

Dem spaßhaften Übergriff und dem ratifizierenden Lachen der Hörer folgt eine rhetorische Frage

die frage isch nadürlich * dio frage isch nadürlich * $o^{\prime \prime} b$ *äh me dəs wirglich nicht hat wissn $\uparrow$ könnan [26-28],

deren Antwort eingespart bzw. durch die folgenden Belege zu inferieren ist.

Die Belegliste bzw. Begründung selbst ist ebenfalls indirekt. Eine darin eingeschobene explizite Perspektivenübernahme und gemeinsame Identitätszuschreibung mit Kritiker 1 aktualisiert dessen ausführliche Belegliste und ermöglicht Kritiker 2 die kurze, übertragene und verallgemeinernde Beweisführung:

dəs (seh=i/) dəs kommt doch do gib i dem kollege lähr völlig re"cht >dos wissot mir ja alts alde hase dəs macht me doch id ousm heitre himml^ * sondern do wird doch vorher dran rumgeboss $/ t \downarrow<[28-30]$.

Die Belege kontrastieren nicht nur kategorial, sondern auch hinsichtlich des StandardDialekt-Kodierniveaus mit den Belegen von Kritiker 1.

Die Forderung im Sinne des ,Zeigefingers' von Kritiker 1 am Ende kann Kritiker 2 sich sparen. Stattdessen folgt eine Nachbearbeitung des parteiwerbenden Übergriffs. Erst hier verdichtet sich wieder der Gebrauch von Formen aus dem Sprechstandardbereich.

\subsection{Vergleich der Redebeiträge}

Im Vergleich der Redebeiträge der beiden Kritiker fallen ein allgemeiner struktureller Zusammenhang sowie wesentliche Kontraste auf: Einerseits doppelt Kritiker 2 die Fallbewertung, den Vorwurf und Begründung von Kritiker 1 in gleichsinniger Weise; dabei setzt er sich aber formal auf verschiedenen Ebenen von den Formulierungen des ersten Redners ab: Kritiker 1 thematisiert und wertet fallbezogen und rekonstruiert den spezifischen Kontext deskriptiv; er argumentiert explizit, detailliert und mit konkreten Referenzen. Dagegen produziert Kritiker 2 in seinem Zweitbezug primär Inferenzstellen, indem er verallgemeinert formuliert, ohne konkrete Referenzen auf Personen oder Funktionen, und in seiner Kategorialität nicht direkt, sondern kontrastiv ist. Die Inferenzgenese basiert dabei auf den Ausführungen des ersten Kritikers, die Kritiker 2 im Zweitbezug als Kontext nutzt.

Die Beziehung der Beiträge hat auch eine kodierniveale Dimension: Die StandardDialekt-Variation ist eingepasst in das grundlegende Strukturierungsmuster, im Zweitbezug Kontrastivität zu produzieren. Am deutlichsten wird der konkomitante StandardDialekt-Kontrast am Beispiel der beiden Beweisführungen, also der standardformgeprägten Belegliste von Kritiker 1, die variativ gedoppelt wird von Kritiker 2, dem die dialektal transformierte Formel ,sowas kommt nicht aus heitrem Himmel' und ,es wird dran rumgebosselt' als Argumente dienen.

Die gleichgerichte Kontrastivität ist mehrfunktional: Beide Einzelbeiträge für sich zeigen die Reliefbildungsfunktion von Variation zur Rede- und Gesprächsordnung, wovon 
ein symbolisches Potential der Sprachformenverwendung oftmals nicht zu trennen ist (bspw. Alfonzetti 1998). Grundsätzlich gilt für die Einzelbeiträge wie für deren Zusammenhang, dass Wiederholung und besonders die variative Wiederholung Nachdruck verschafft und als Formulierungsarbeit am Geltungsbereich und Wahrheitsanspruch der Äußerung zu sehen ist. Kritiker 2 schlägt durch das Kontrastverfahren bei der Rekontextualisierung der Meinung von Kritiker 1 zudem nicht nur kollaborativ in die gleiche Kerbe, sondern bleibt dabei als eigenständiger Sprecher mit eigenem Verhältnis zum Sachverhalt erkennbar.

Die Einzelbeiträge wie auch deren Zusammenhang verdeutlichen, dass Sprachvariation von den Sprechern kompetent als sprachliches Mittel neben andern zur Bearbeitung kommunikativer Aufgaben und zur Unterstützung ihrer rhetorischen Verfahren verwendet wird. Sprachvariation ist nicht fest an das Thema oder globale Faktoren der Redesituation gekoppelt, sondern wird flexibel in Bezug auf interaktionsstrukturelle Anforderungen und Gegebenheiten des aktuellen Gesprächstands gebraucht.

\section{Schluss}

Das Beispiel sollte deutlich machen, dass zwischen der Interaktionsstruktur und der Variationsstruktur Relationen bestehen. Sprachvariation ist interaktionsstrukturell fundiert und die Analyse von Sprachvariationsverfahren - insbesondere funktionaler Aspekte setzt letztlich die Analyse der Interaktionsstruktur voraus.

Bei der Interaktionsstrukturanalyse lässt sich zurückgreifen auf Konzepte von Werner Kallmeyer. Das grundlegende Analysekonzept ist das der Interaktionskonstitution. Betrachtet man die Variationsverfahren und deren interaktionale Funktionen, kommt die konkrete Formulierung der einzelnen Sprecher und damit der Prozess der Konstitution der Äußerungsbedeutung in den Blick. Die Äußerungskonstitution hängt eng mit der Interaktionskonstitution zusammenhängt, sie verläuft quer zur Interaktionskonstitution ebenso wie gesprächsrhetorische Aspekte (Kallmeyer 1996).

Bei der Bearbeitung aller Ebenen der Interaktionskonstitution kann Sprachvariation als Mittel verwendet werden. Im präsentierten Ausschnitt der rhetorisierten Kommunikation betrifft die Variation des Kodierniveaus insbesondere die Interaktionskonstitutionsebene der Sachverhaltsdarstellung. Die Verfahren helfen, die Äußerungen zu kontextualisieren, konturieren und/oder zu modalisieren - im besprochenen Beispiel primär, um am Geltungsbereich der Darstellung zu wirken.

Sprecher behandeln die einzelnen Interaktionskonstitutionsebenen selten getrennt voneinander. Wie im Beispielausschnitt ist die Ebene der Identität und Beziehung in vielen Fällen mehr oder weniger immer mitbehandelt: Implizit geht es im Beispiel bei jeder Äußerung um das, was Kritiker 2 bei der Identitätszuschreibung (dəs wissət mir ja alts alde hase) expliziert, nämlich um die Selbstpositionierung als Politiker, die wissen, wie solche dinge laufen.

Das Beispiel zeigt, wie Sprachvariation bei der Bearbeitung der oft zusammenhängenden Konstitutionsebenen Identität/Beziehungen und Interaktionsmodalität einsetzbar ist - insbesondere hinsichtlich der Kooperationsform zwischen den Teilnehmern. Man bedenke hierbei, dass die Gemeinderäte ihre Äußerungen kalibrieren müssen hinsichtlich des beabsichtigten eigenen politischen Kapitals und der gleichzeitigen Risiken, nämlich die grundlegende Kooperationsbasis und Beziehungen nicht wirklich zu gefährden. Der 
Zusammenhang von Variationsverfahren mit Beziehungsarbeit und der Interaktionsbzw. Äußerungsmodalität wird am deutlichsten am Beispiel des Übergriffs von Kritiker $2^{22}$, bei dem u.a. durch Codeswitching in den Basisdialektbereich kontextualisiert wird, dass seine Äußerung in einer gewechselten Modalität, nämlich der des doppelbödigen Spaßes zu interpretieren ist.

\section{Literatur}

Alfonzetti, Giovanna (1998): The conversational dimension of code-switching between Italian and dialect in Sicily. In: Auer, Peter (Hg.): Code-Switching in Conversation: Language, Interaction and Identity. London. S. 27-48.

Alvarez-Cáccamo, Celso (1990): Rethinking conversational code-switching: Codes, speech varieties, and contextualization. In: Hall, Kira/Koenig, Jean-Pierre/Meacham, Michael/Reinman, Sondra/Sutton, Laurel A.: Proceedings of the 16. Annual Meeting of the Berkeley Linguistics Society. General Session and Parasession on the Legacy of Grice. Berkeley. S. 3-16. URL: www.udc.es/dep/ lx/cac/ artigos/bls90.pdf, Stand 2004 .

Auer, Peter (1984): On the meaning of conversational code-switching. In: Auer, Peter/Di Luzio, Aldo (Hg.): Interpretive sociolinguistics. Tübingen. S. 87-108.

Auer, Peter (1986): Konversationelle Standard/Dialekt-Kontinua (Code-Shifting). In: Deutsche Sprache 14, S. 97-124.

Auer, Peter (1992): Introduction: John Gumperz’ Approach to Contextualization. In: Auer, Peter/Di Luzio, Aldo (Hg.): The Contextualization of Language. Amsterdam. S. 1-37.

Auer, Peter (1995): The Pragmatics of Code-switching: A Sequential Approach. In: Milroy, Lesley/Muysken, Pieter (Hg.): One Speaker, Two Languages. Cross-disciplinary Perspectives on Codeswitching. Cambridge. S. 115-135.

Auer, Peter (Hg.) (1998): Code-Switching in Conversation: Language, Interaction and Identity. London.

Auer, Peter (1999): From code-switching via language mixing to fused lects: toward a dynamic typology of bilingual speech. In: International Journal of Bilingualism 3, 4. S. 309-332.

Auer, Peter (2004): Europe's sociolinguistic unity, or: A typology of European dialect/standard constellations. In: Delbecque, Nicole/van der Auwera, Johan/Geeraerts, Dirk (Hg.): Perspectives on Variation. Berlin/New York. S. 7-42.

Bellmann, Günter (1983): Probleme des Substandards im Deutschen. In: Mattheier, Klaus (Hg.): Aspekte der Dialekttheorie. Tübingen. S. 105-130.

Bellmann, Günter (1997): Between Base Dialect and Standard Language. In: Folia Linguistica 32, 1-2. S. 23-34.

Berruto, Gaetano (1987): Varietät. In: Ammon, Ulrich [u. a.] (Hg.): Soziolinguistik. Ein internationales Handbuch zur Wissenschaft von Sprache und Gesellschaft. 1. Teilbd. (=HSK 3.1). Berlin/New York. S. 263-267.

Bilmes, J. (1991): Toward a theory of argument in conversation: The preference for disagreement. In: van Eemeren, Frans. H./Grootendorst, Rob/Blair, J. Anthony/Willard, Charles A. (Hg.): Proceedings of the Second International Conference on Argumentation. Amsterdam: International Centre For The Study Of Argumentation. S. 461-469.

Cook-Gumperz, Jenny/Gumperz, John (1976): Context in children's speech. In: Cook-Gumperz, Jenny/Gumperz, John: Papers on Language and Context. Berkeley. (=Working Paper No 46).

\footnotetext{
${ }^{22}$ Siehe Ereignisfläche 25-26 im Transkript bzw. die Analyse auf Seite 9.
} 
Deppermann, Arnulf (2000): Ethnographische Gesprächsanalyse: Zum Nutzen einer ethnographischen Erweiterung für die Konversationsanalyse. Gesprächsforschung 1. S. 96-124. URL: http://www. Gespraechsforschung-ozs.de/heft2000/ga-deppermann.pdf. Stand 2005.

Dieckmann, Walther (1981): Inszenierte Kommunikation. Zur symbolischen Funktion kommunikativer Verfahren in (politisch-)institutionellen Prozessen. In: Dieckmann, Walther: Politische Sprache - politische Kommunikation. Vorträge, Aufsätze, Entwürfe. Heidelberg. S. 255- 279.

Gilles, Peter (2003): Zugänge zum Substandard: Korrelativ-globale und konversationell-lokale Verfahren. In: Androutsopoulos, Jannis/Ziegler, Evelyn (Hg.): Standardfragen. Frankfurt am Main. S. 195-217.

Gumperz, John (1982): Discourse Strategies. Cambridge.

Hinnenkamp, Volker (1987): Foreigner talk, code-switching and the concept of trouble. In: Knapp, Karlfried/Enninger, Werner/Knapp-Potthoff, Annelie (Hg.): Analyzing Intercultural Communication. Berlin/New York. S. 137-180.

Jefferson, Gail (1991): List construction as a task and resource. In: Psathas, George (Hg.): Interactional Competence. New York. S. 63-92.

Kallmeyer, Werner (1978): Fokuswechsel und Fokussierungen als Aktivitäten der Gesprächskonstitution. In: Meyer-Hermann, Reinhard (Hg.): Sprechen - Handeln - Interaktion. Ergebnisse aus Bielefelder Forschungsprojekten. Tübingen. S. 179-250.

Kallmeyer, Werner (1982): Aspekte der Analyse verbaler Interaktion - Zwischenbilanz eines Forschungsprogramms. Unveröffentlichter Text. Mannheim.

Kallmeyer, Werner (Hg.) (1994): Kommunikation in der Stadt. Teil 1. Exemplarische Analysen des Sprachverhaltens in Mannheim. Berlin/New York.

Kallmeyer, Werner (1996): Einleitung. In: Kallmeyer, Werner (Hg.): Gesprächsrhetorik. Tübingen. S. 719.

Kallmeyer, Werner (1996b): Unser schbroch is a e schbroch. In: Sprachreport, 2/1996. S. 14-16.

Kallmeyer, Werner/Keim, Inken (1994): Phonologische Variation als Mittel der sozialen Symbolisierung in der Filsbachwelt. In: Kallmeyer, Werner (Hg.): Exemplarische Analysen des Sprachverhaltens in Mannheim. Berlin/New York. S. 141-249. (=Komm. i. d. Stadt 4.1.).

Kallmeyer, Werner/Keim, Inken/Nikitopoulos, Pantelis (1982): Zum Projekt „Kommunikation in der Stadt“. In: Bausch, Karl-Heinz (Hg.) (1982): Mehrsprachigkeit in der Stadtregion. Düsseldorf. S. 345390. (= Sprache der Gegenwart Bd. 56, Jahrbuch 1981 des Instituts für deutsche Sprache).

Keim, Inken (1995): Kommunikative Stilistik einer sozialen Welt „kleiner Leute“ in der Mannheimer Innenstadt . Berlin/New York. . (=Kommunikation in der Stadt 4.3.).

Knöbl, Ralf (i.V.): Sprachvariation in einer schwäbischen Kleinstadt. Varietäten im Diskurs. Dissertationsprojekt Uni. Mannheim.

Knöbl, Ralf (i. Dr.): Binnensprachliche Variation: Code-Switching und Mixing im Schwäbischen. In: Wolff, Dieter: Mehrsprachige Individuen - vielsprachige Gesellschaften. Frankfurt am Main. S. 59-86.

Kohler, Klaus J. (1995²): Einführung in die Phonetik des Deutschen. Berlin.

Kühn, Peter (1995): Mehrfachadressierung. Untersuchungen zur adressatenspezifischen Polyvalenz sprachlichen Handelns. Tübingen.

Lameli, Alfred (2004): Dynamik im oberen Substandard. In: Scheuringer, Hermann/Gaisbauer, Stephan (Hg.): Linzerschnitten. Beiträge zur 8. Bayerisch-österreichischen Dialektologentagung. Linz. S. 197208.

Lenz, Alexandra (2003): Struktur und Dynamik des Substandard. Eine Studie zum Westmitteldeutschen. Wiesbaden. Beiheft 125 der ZDL.

Luckmann, Benita (1970): Politik in einer deutschen Kleinstadt. Stuttgart. 
Mihm, Arend (2000): Die Rolle der Umgangssprachen seit Mitte des 20. Jahrhunderts. In: Besch, Werner/Betten, Anne/Reichmann, Oskar/Sonderegger, Stefan (Hg.): Sprachgeschichte. Ein Handbuch zur Geschichte der deutschen Sprache und ihrer Erforschung. 2. Auflage. Bd. 2. Berlin/New York. S. 2107-2137.

Myers-Scotton, Carol (1993): Social Motivations of Code-Switching. Oxford.

Sacks, Harvey (1987): On the preferences for agreement and contiguity in sequences in conversation. In: Button, Graham/Lee, J.R.E. (Hg.): Talk and Social Organization. Cleveland/ Philadelphia.

Schmidt, Jürgen Erich (2005): Versuch zum Varietätenbegriff. In: Lenz, Alexandra/Mattheier, Klaus (Hg.): Varietäten - Theorie und Empirie. Frankfurt am Main. S. 61-74.

Sebba, Mark/Wootton, Tony (1998): We, they and identity: Sequential vs. identity-related explanation in code-switching. In: Auer, Peter (Hg.): Code-Switching in Conversation: Language, Interaction and Identity. London. S. 262-286.

Selting, Margret (2004): Listen: Sequenzielle und prosodische Struktur einer kommunikativen Praktik eine Untersuchung im Rahmen der interaktionalen Linguistik. In: Zeitschrift für Sprachwissenschaft 23, S. 1-46.

Schwitalla, Johannes (1995): Kommunikative Stilistik zweier sozialer Welten in Mannheim-Vogelstang. Berlin/New York. (=Kommunikation in der Stadt 4.4.).

Wei, Li (1998): The 'why' and 'how' questions in the analysis of conversational code-switching. In: Auer, Peter (Hg.): Code-Switching in Conversation: Language, Interaction and Identity. London. S. 156-176.

\section{Anhang}

\section{Transkriptionskonventionen (DIDA)}

Es wird eine Partiturschreibweise verwendet. Simultane Äußerungen stehen übereinander.

$\begin{array}{ll}* & \text { kurze Pause (bis max. 1/2 Sekunde) } \\ * * & \text { etwas längere Pause (bis max. 1 Sekunde) } \\ * 3,5^{*} & \text { längere Pause mit Zeitangabe in Sekunden } \\ = & \text { Verschleifung (Elision) bes. bei klitischen Verbindungen } \\ / & \text { Wortabbruch } \\ (\ldots) & \text { unverständliche Sequenz } \\ (\mathrm{xxx}) & \text { vermuteter Wortlaut } \\ \uparrow & \text { steigende Intonation (äußerungsfinal) } \\ \downarrow & \text { fallende Intonation (äußerungsfinal) } \\ - & \text { schwebende Intonation } \\ " & \text { auffällige Betonung, meistens Satzakzent } \\ : & \text { auffällige Dehnung } \\ \rightarrow \mathrm{xxx} \leftarrow & \text { schneller (relativ zum Kontext) } \\ \leftarrow \text { xxx } \rightarrow & \text { langsamer (relativ zum Kontext) } \\ >\mathrm{xxx}< & \text { leiser (relativ zum Kontext) } \\ <\mathrm{xxx}> & \text { lauter (relativ zum Kontext) } \\ \text { LACHT } & \text { Sprechern zuordenbare, nichtmorphemisierte Äußerungen (in Sprecherzeile) } \\ \text { LACHEN } & \text { Kommentare zur Äußerung, sonstige akustische Ereignisse in der Gesprächssituation } \\ & \text { sowie nicht zuordenbare nichtmorphemisierte Äußerungen in der globalen Kommentar- } \\ & \text { zeile (K\&) }\end{array}$




\section{Transkripte:}

Erstbezug (Kritiker 1):

[1]

[2]

\begin{tabular}{|l|l|l|l|}
\hline K1: & (ja) also i find ne to"lle leistung war dəs nicht $\uparrow$ liebe verwalt ${ }^{\text {ung }} \uparrow$ & & uns hier- * \\
\hline K\&: & & LEISES LACHEN & \\
\hline
\end{tabular}

\begin{tabular}{|l|l|}
\hline K1 : & äh n vorschlag zu machn gebührən zu senken- * un am gləIchṇ tag beschließt- * äh də \\
\hline
\end{tabular}

[3]

\begin{tabular}{l|l}
\hline K1 : & greistagsousschuss * äh erhöhte kostṇ $\uparrow$ und anscheinend nix dəvon zu wi"ssṇ $\downarrow$ also da frag
\end{tabular}

[4]

\begin{tabular}{|c|c|c|}
\hline K1: & I mI scho $\uparrow^{*}$ fü was he=mə einlich en & $\begin{array}{l}\text { bürgermei schder im greisdag sitzṇ } \uparrow^{*} \text { äh der muss } \\
\text { STÖRUNG }\end{array}$ \\
\hline
\end{tabular}

[5]

\begin{tabular}{|l|l|l|l|l|}
\hline K1: & doch informiert sein & $\begin{array}{l}\text { über } \\
\text { STÖRUNG }\end{array}$ & solche di"nge- dəs würd i einfach erwardṇ $\uparrow$ & $\begin{array}{l}\text { drum- * >si=mə ja } \\
\text { STÖRUNG }\end{array}$ \\
\hline K\&: &
\end{tabular}

[6]

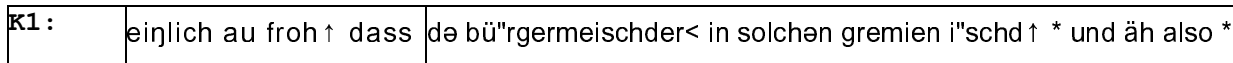

[7]

\begin{tabular}{|l|l|}
\hline K1: & so"lche dinge die gfallət mir ü"berhaubt nicht $\uparrow$ muss i scho sagṇ- dəs isch au ne- * koI
\end{tabular}

[8]

\begin{tabular}{|l|l|l}
\hline K1 : & übermäßig tolle leischdung $\downarrow$ * äh denn- $\mathrm{m} \rightarrow \mathrm{i}$ moIn $\leftarrow$ solche dinge ** äh ä da müssn \\
\hline
\end{tabular}

[9]

\begin{tabular}{|l|l|}
\hline K1 : & vo"rinformationen dasein- də äh landrats/ oder də landkreis- * der äh * macht dəs ja nicht so" \\
\hline
\end{tabular}

[10]

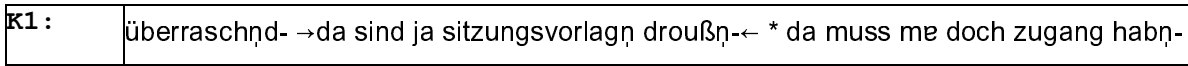

[11]

[12]

\begin{tabular}{|l|l|}
\hline K1: & insbesondre die verwaltung muss zu solchən dingn zugang habn $\downarrow$ * je"tzt $\uparrow$ bləIbt uns \\
\hline
\end{tabular}

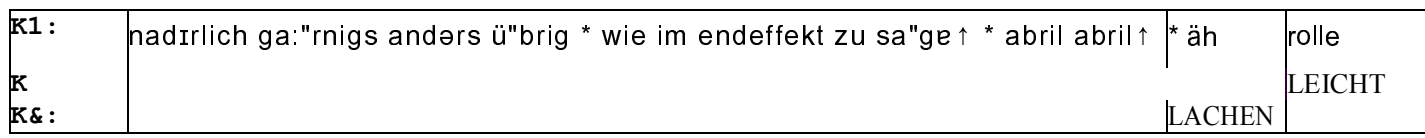

[13]

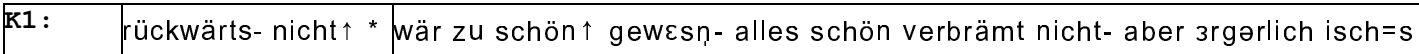
K SCHMUNZELND

[14]

\begin{tabular}{l|l}
\hline K1: & dro"tzdem $\downarrow$ * und im endeffekt äh sollt sowas also * wIrglich e əI"nmaligə gschichde bləIbe \\
\hline
\end{tabular}

[15]

\begin{tabular}{|l|l|}
\hline K1: & >un=dann würd i orfa bi"ttn- $-<{ }^{*}$ wenn solche dinge si"nd $\uparrow{ }^{*}$ dass äh die hərrən kreis $\uparrow /$ und \\
\hline
\end{tabular}

[16]

K1: $\quad$ da"mən- * greisräte $\rightarrow$ aber insbesondre do drifft=scho sie- $\leftarrow$ * alts də bürgərmeischdər * au im

[17]

\begin{tabular}{|l|l|l|l}
\hline K1: & kreisrat nicht $\uparrow$ aber * äh einen wo mir einlich erwa"rtṇ $\uparrow$ dass die information dort a"nkomm * \\
\hline
\end{tabular}

[18]

\begin{tabular}{|l|l|l|}
\hline K1: & ähm dass so"lche dinge ei"nmalig bleibən $\downarrow^{* *}$ & \\
\hline K\&: & WASSERGLAS & {$[\ldots] 2: 30$ MINUTEN AUSGELASSEN } \\
\hline
\end{tabular}




\section{Zweitbezug (Kritiker 2):}

[19]

[20]

\begin{tabular}{|l|l|}
\hline K2: & isch sa"chlich sicher richdich- wenn man die bəItragsstruktur- ${ }^{*}$ də koshdnstruktur anpasst- \\
\hline
\end{tabular}

[21]

\begin{tabular}{|l|l}
\hline K2: & sie hent grad die * äh fix und variablən koshdṇ ja $\uparrow{ }^{*}$ da angeführt $\uparrow{ }^{* *}$ äh də \\
\hline
\end{tabular}

[22]

\begin{tabular}{l|l}
\hline K2: & vəwaldungsvorschlag ** isch sicher unumgenglich ** äh und * deng es gibt koI große
\end{tabular}

[23]

[24]

[25]

[26]

\begin{tabular}{|l|l|l|l|}
\hline K2: & wenn d frəIe wehler koI grei"sdagsmandat hent $\downarrow$ & aber (dann) die frage isch nadürlich * diə
\end{tabular} K\&: $\quad$ LACHEN

[27]

\begin{tabular}{l|l|l|l|l|l}
\hline K1: & die wärət wahrschei lich z=spät komme & \\
K2: & frage isch nad & ürlich * & me dəs wirglich nicht hat wissṇ $\uparrow$
\end{tabular}

[28]

[29]

\begin{tabular}{|l|l|l|l|}
\hline K2: & könnən dəs $($ seh=i/) dəs kommt doch & $\begin{array}{l}\text { do gib I dem } \\
\text { SCHNÄUZEN }\end{array}$ & kollege lähr völlig re"cht >dəs wissət mir ja \\
\hline K\&: &
\end{tabular}

[30]

K2: $\quad$ alts alde hase dəs macht me doch id ousm heitre himml $\uparrow{ }^{*}$ sondern do wird doch vorher

K\&:

LACHEN

\begin{tabular}{|l|l|l|l}
\hline K2: & dran rumgeboss $\mid t \downarrow<{ }^{*}<$ guət> es $\mathrm{i}=$ scho so $\downarrow$ etzt äh müsse mir reagiere- * und äh * sie ham \\
\hline
\end{tabular}

[31]

[32]

\begin{tabular}{|l|l|l|l|l|l|l|l}
\hline K2: & äh məInən bər"trag- * nicht als * a"nmaßnt $t^{\text {h }}$ & $\begin{array}{l}\text { empfunde mit də frəIe wehler (sondern erkannt) } \\
\text { LACHEN }\end{array}$ \\
\hline K\&: & & \\
\hline
\end{tabular}

\begin{tabular}{l|l|l|l|l|}
\hline BM: & LACHT & frau maurer ** & \\
K2: & dass des also e spass au"ch & sein sollt & e bissle nebeher * (sicher) & $\mid$ i muss=etz do \\
FH : & K\&: & & $\mid$ \\
\hline
\end{tabular}

Ralf Knöbl M.A.

Institut für Deutsche Sprache

Postfach 101621

D-68016 Mannheim

e-mail:knoebl@ids-mannheim.de 
Zur interaktionsstrukturellen Fundiertheit von Sprachvariation 